\title{
High-Resolution Electron Microscope Structure Images of Metal Particles
}

\author{
Jan-Olov Bovin* and Jan-Olle Malm
}

National Center for HREM, Inorganic Chemistry 2, Chemical Center, University of Lund, P.O. Box 124, S-221 00 Lund, Sweden

\begin{abstract}
Bovin, J.-O. and Malm, J.-O., 1991. High-Resolution Electron Microscope Structure Images of Metal Particles. - Acta Chem. Scand. 45: 791-796.

Dynamic events on the surfaces and in the bulk of small metal particles have been recorded with a low light level TV camera mounted on a high-resolution transmission electron microscope (HRTEM) with the structural resolution of $0.16 \mathrm{~nm}$. The dynamic structure of small metal crystals, in this case $\mathrm{Au}$ and $\mathrm{Ag}$, can be classified under three headings: rearrangements of the total crystal structure; "clouds" of atoms existing outside surfaces, involved in growth and rearrangements of the surface structure; and localized atom hopping during crystal growth. Experimental images of five-fold particles are compared to simulated ones of two different models with five-fold symmetry.
\end{abstract}

Dedicated to Professor Sten Andersson on the occasion of his 60th birthday.

The atomic structure of clusters, including those with a few tens up to many thousands of atoms, is of great importance, since their physical and chemical properties are strongly dependent on the atomic arrangements. The stability with respect to time and to reactions in different environments is also of great interest. Even if the basic studies of cluster structures are still in their initial stages, their practical use is already important in the areas of catalysis, metal deposition and metal powder techniques. Future developments in, for example, laser techniques and magnetic discs may benefit from their special electrical, magnetic and optical properties.

As a consequence of technical improvements, high-resolution transmission electron microscopes (HRTEMs) are nowadays capable of atomic imaging. The use of low-light TV cameras, together with modern videorecording techniques, has made it possible to study dynamic events in small metal particles with diameters of one to several tens of nanometres. Under circumstances where the particles are thin enough (for most particles this is the case at the edges), and provided the surface is devoid of impurities, the HRTEM image shows the crystal surface profile structure. If the microscope is capable of atomic resolution, this profile reveals the projected arrangement of atomic columns along the surface, so-called surface profile images.

Evaporated small gold clusters, down to $50 \AA$ in size, have previously been studied by TEM, and images revealing planar defects and twinning were published by Ino. ${ }^{1}$ The atomic structure of clusters viewed along [110] was not resolved until Cosslet et al. ${ }^{2}$ imaged gold particles showing atomic columns using a $600 \mathrm{kV}$ HREM. The same in-

\footnotetext{
* To whom correspondence should be addressed.
}

strument was used for recording the first surface profile images of gold atomic columns. ${ }^{3}$ The total structural rearrangement of small gold clusters was discovered by Bovin, ${ }^{4}$ and with use of a low-light TV system, including TV rate image processing, it was possible to record crystal growth and atomic structure changes in real time. ${ }^{5}$ The dynamic behaviour of small metal clusters was verified later by Iijima and Ichihashi ${ }^{6}$ and high-vacuum studies by HREM have shown that the structural rearrangements are dependent on temperature. ${ }^{7,8}$

A serious problem when studying small clusters is their instability when transferred in air to the microscope column. The last decade of developments by Schmid et al. ${ }^{9,10}$ in preparing air-stable clusters in solution have solved that problem in some cases. These so-called Schmid clusters of transition and noble metals with stabilising organic ligands are useful for HRTEM, since the electron beam will desorb the ligands into the microscope vacuum and a "clean" metal surface can be profile imaged.

\section{Experimental}

The metal clusters used in this investigation originate from the cluster molecule ${ }^{9} \mathrm{Au}_{55}\left(\mathrm{PPh}_{3}\right)_{12} \mathrm{Cl}_{6}$ and from a stabilised silver particle sample. The organic ligands and the chlorine atoms are evaporated from the metal core of the cluster when irradiated by an electron beam flux of $(10-25) \times 10^{3}$ electron $\AA^{-2} \mathrm{~s}^{-1}$. The metal clusters left are randomly oriented on the amorphous carbon support and are best imaged sitting on the edge of the holes of the support.

The HRTEM work was performed with a JEM-4000EX instrument equipped with a high-resolution pole piece $\left(C_{\mathrm{s}}=1 \mathrm{~mm}\right)$ operated at $400 \mathrm{kV}$. The vacuum in the speci- 
men chamber is at best $5 \times 10^{-6} \mathrm{~Pa}$. The structural resolution was determined by optical diffraction of a micrograph of amorphous carbon to be $0.16 \mathrm{~nm}$ from the first zero of the contrast transfer function at Scherzer focus. A TVsystem, Gatan 622 , is connected by optical fibres to the bottom of the microscope viewing chamber. Video-rate image processing for noise reduction, contrast enhancements and frame grabbing was performed with a digital video processor, Quantex DS-30. Image simulations, using the microscope optical parameters, were computed with an updated local version of the SHRLI programs. ${ }^{\prime \prime}$

\section{Results and discussion}

Dynamics of small crystals, such as $\mathrm{Au}, \mathrm{Ru}, \mathrm{Pt}, \mathrm{Pd}, \mathrm{Rh}, \mathrm{Ag}$ and $\mathrm{Pb}$, can be classified under three headings: rearrangements of the total crystal structure; "clouds" of atoms existing outside surfaces, involved in growth and rearrangements of the surface structure; and localized atom hopping during crystal growth. Differences in behaviour depend on the element involved, particle size, method of preparation, substrate, degree of contact with the support and the beam current density.

Rearrangements of the total crystal structure. The occurrence of rapid structural rearrangements is one of the most captivating properties of small metal clusters. It has been found in, for example, Au, ${ }^{5-8} \mathrm{Pt},{ }^{12} \mathrm{Rh},{ }^{13} \mathrm{Ru},{ }^{14}$ and Ag. ${ }^{15}$ The general tendencies are that the smaller the clusters are, the faster the structural transformations will occur. The smaller the contact area with the support is, the faster the structure will change. Most observations ${ }^{7,8}$ so far indicate a temperature dependence of all structural transformations.

It has been shown ${ }^{14}$ that during an observation at high constant beam flux $\left(25 \times 10^{3}\right.$ electron $\left.\AA^{-2} \mathrm{~s}^{-1}\right)$ a metal cluster can change the total structure from hexagonal close packing (h.c.p.) to face-centred cubic close packing (c.c.p.), next to body-centred cubic packing (b.c.c.), and finally to the fourth well known packing of small metal clusters, so-called icosahedral packing of atoms. In this case there is no doubt that the cluster is changing its total structure. During the observation of dynamic events in metal clusters, the clusters sometimes show no structural periodicity. This can be explained in three ways. Either the crystal is transformed so fast that one TV frame (1/25 s) will average diffuse information from several structures, or the cluster is rotating fast on the support. The third possibility is the occurrence of a molten phase of the cluster. At a moderate beam intensity the temperature is raised ${ }^{8}$ less than $100^{\circ} \mathrm{C}$, meaning that the molten stage would only occur at very high beam intensities or if the whole stage were heated from the outside. The presence of different structure types in the same particles during observations with constant beam flux indicates a very narrow range of energy levels of stability for the different structure types. This has been postulated from theoretical calculations by Ajayan and Marks. ${ }^{16}$ It is well known that small impurities on a surface will strongly effect the surface diffusion, and because small clusters are greatly dominated by surface atoms, the residual gas molecules in the microscope may effect the rate of transformation. Further experiments with controlled leakage of a known gas into the specimen chamber are necessary.

Rotation of the cluster on the support is commonly observed, and this is illustrated in the video images recorded of the three different projections of the same icosahedral gold cluster in Fig. 1. The first image captured, Fig. 1(a), shows the cluster close to the five-fold direction, and the second image, Fig. 1(b), recorded a few seconds later, reveals the three-fold symmetry; later the two-fold symmetry can be seen [Fig. 1(c)].

To establish exactly the atomic structure of the icosahedral small clusters from HREM is a difficult task.
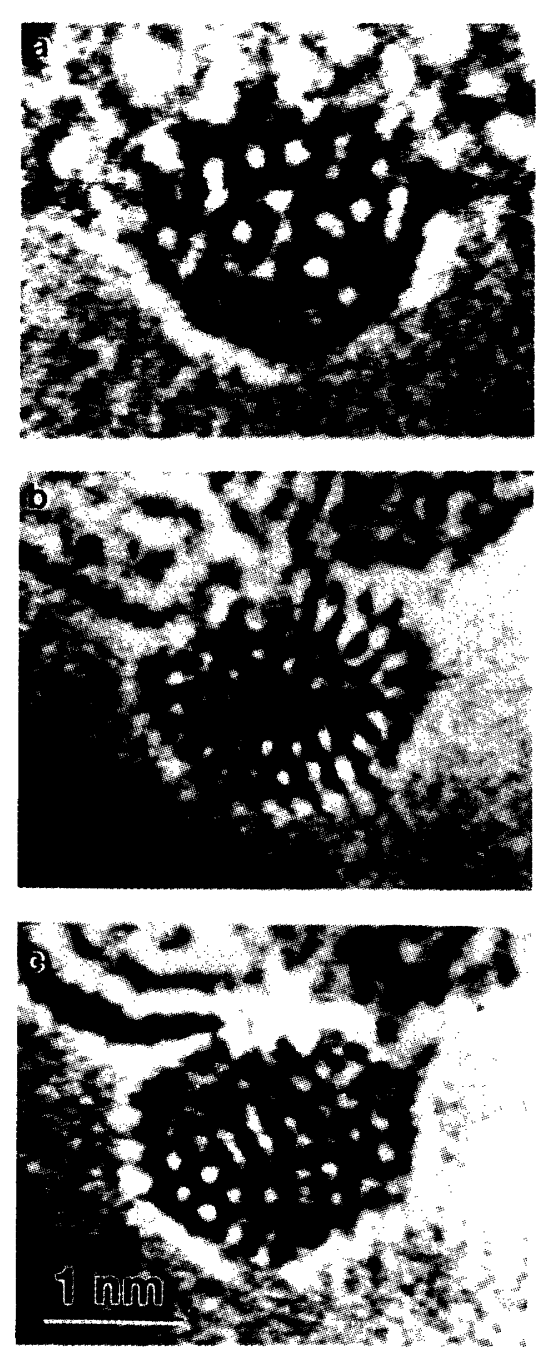

Fig. 1. Single-frame video tape HRTEM images of a small gold cluster rotating on an amorphous carbon support. The cluster is ca. $2 \mathrm{~nm}$ in diameter with around 400 atoms. The icosahedral structure is imaged with near five-fold symmetry in (a) and with three-fold symmetry in (b). The typical two-fold symmetry can be seen in (c). 

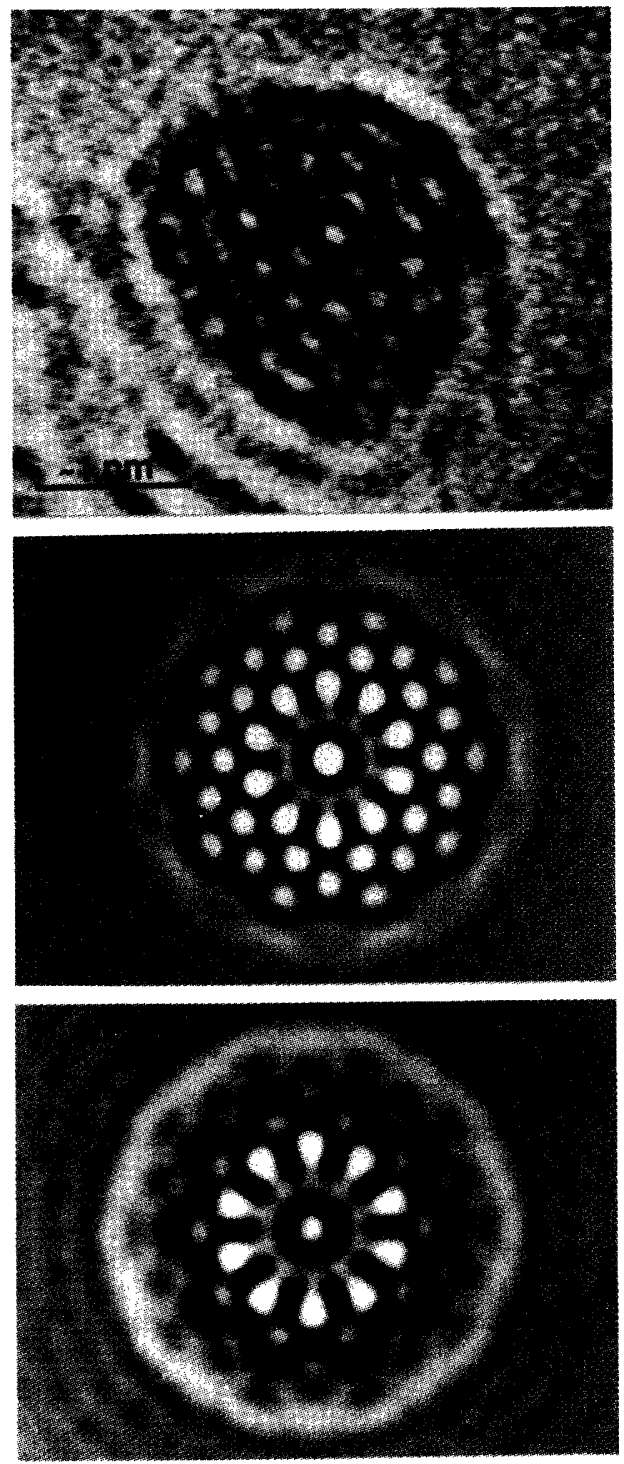

Fig. 2. Experimental image of a gold particle showing five-fold symmetry (a) compared to two simulated models (b and c). Model (b) is an icosahedron (309 atoms) viewed along the five-fold direction, and model (c) is a building block taken from a structural model of a quasi-crystal (slightly smaller than the icosahedron). The simulations are made under optimum conditions with the electro-optical parameters of the JEM $4000 E X$ used and with an underfocus of $-76 \mathrm{~nm}$. Both models were placed in a super-cell with the dimensions $4 \mathrm{~nm} \times 4 \mathrm{~nm} \times 2.3 \mathrm{~nm}$ and were viewed along the short axis. The simulations were made with a sampling array of $128 \times 128$ points. The comparison shows that even under optimum conditions it would be difficult to link the experimental image to either of the two models.

Preliminary image simulations computed with the SHRLI programs using the icosahedral model of packing atoms and, in comparison, a model with five-fold conformal symmetry taken from a structural model of a quasicrystal, ${ }^{17}$ show that it will be complicated to distinguish between these two models even under optimum conditions (Fig. 2).

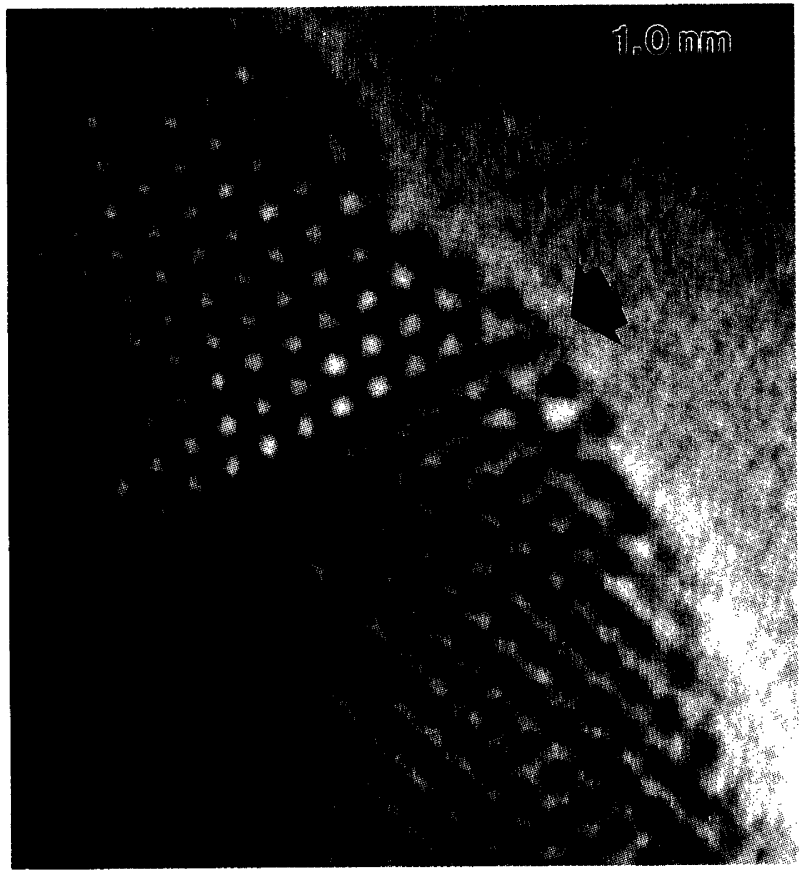

Fig. 3. An example of a (111) twin plane (arrowed) found in a metal particle. This image is from a silver particle viewed along the [110] direction.

It should also be taken into account that the images of five-fold particles are very sensitive to the orientation of the particle.

The concept of chemical twinning ${ }^{18.19}$ has played an important role in the understanding of structural relations and defects. The geometrical relationships in (111) $)_{\mathrm{fcc}}$ twinning, for example, have been known for a long time. ${ }^{20}$ These features are common in small metal particles (Fig. 3). Local transformations often occur in small clusters; (111) twin planes are common in c.c.p. clusters and they can disappear on going from one TV frame to another (cf. Fig. 4). The presence of (111) twin planes does not alter the packing efficiency of the crystal, but merely introduces one unit cell of h.c.p. If the twin planes are introduced with an alternating regular repeat the crystal will change from c.c.p. to h.c.p., and clusters of half c.c.p. and half h.c.p. are not uncommon. A stepwise disappearance of a twin plane in a gold cluster is illustrated in Fig. 4. The (111) twin plane marked with an arrow in Fig. 4(a) is dividing the cluster into two c.c.p. parts. By changing the atomic positions of the two upper layers [marked with arrowheads in (b)] in the first step and finally changing the positions of the three top layers [marked with arrowheads in (c)] along the same vector the whole cluster is transformed into c.c.p.

"Clouds" of atoms. Off-lattice dynamical events outside surfaces of clusters have been reported from HRTEM work by several research groups. The existence of diffuse, varying contrast extending up to $1 \mathrm{~nm}$ from the surfaces was first found outside the $\{100\},\{110\}$ and $\{331\}$ surfaces 

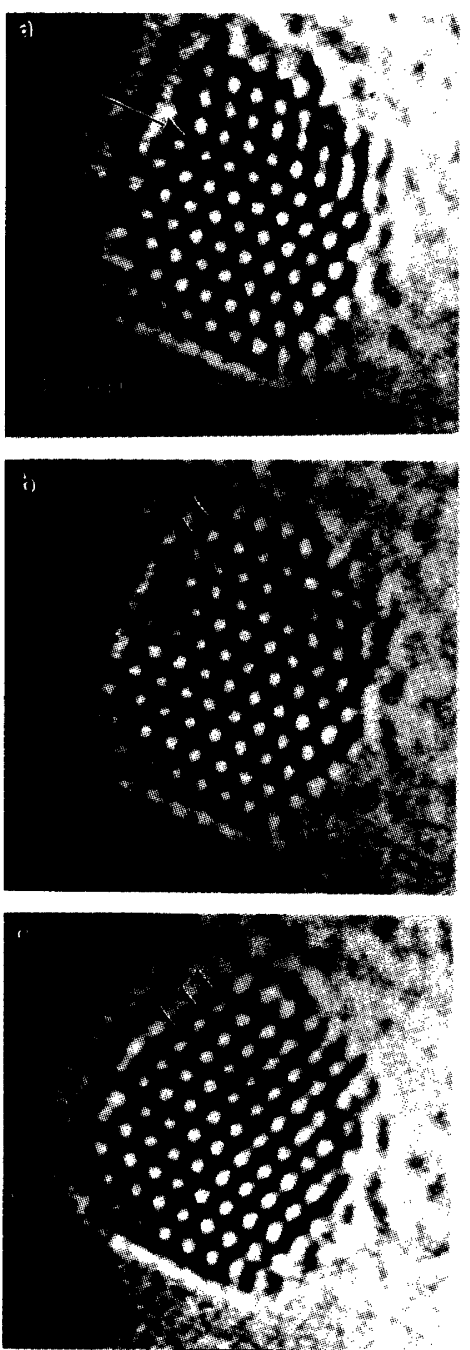

Fig. 4. Stepwise disappearance of (111) twinning in a gold cluster. The initial stage with its twin plane is marked with an arrow in (a) and one TV frame (1/25 s) later the two top layers [arrowheads in (b)] of the cluster have changed orientation in period with the base of the crystal. Finally all three top layers [arrowheads in (c)] have changed positions so the total cluster is c.c.p.

of gold by Bovin et al. ${ }^{21}$ and Iijima. ${ }^{22}$ Lately it has also been found, ${ }^{23}$ using ultra-high-vacuum HRTEM, to exist between two small gold clusters supported on carbon. The nature of the "clouds" of atoms outside the surfaces of metals has not so far been fully understood, because normal chemical bonds to the surface can not explain the existence of atoms at such a long distance from the surface. It has been suggested that the cloud may be due to surface contaminations, but lately UHV studies ${ }^{23}$ make that appear unlikely. The observation of atomic clouds with fluctuating contrast is always combined with extensive changes in the occupancy of the surface atom positions. A probable explanation of this can be found in partial surface melting, suggested from theoretical molecular-dynamic simulations for c.c.p. aluminium. ${ }^{24}$ From such calculations it is found
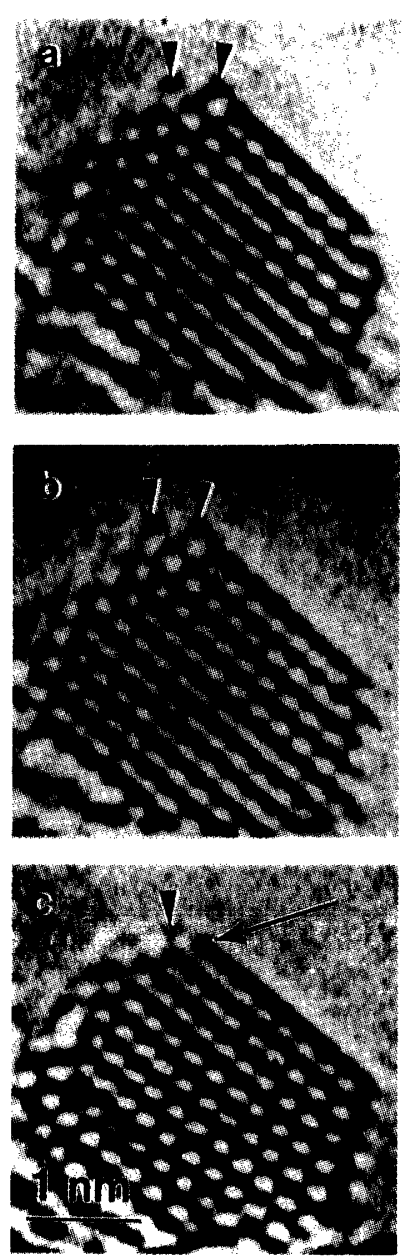

Fig. 5. Growth of a $\{100\}$ surface layer on a c.c.p. gold cluster. The images in (a) and (b) show the appearance of a somewhat ordered "cloud" of atoms (marked with arrowheads) prior to the ordering of the new surface [marked with a long arrow in (c)]. A new "cloud" [marked with an arrowhead in (c)] can be seen before the next layer starts to order. The video sequence is recorded within a few seconds.

that the surface region of $\{110\}$ starts to melt about $200 \mathrm{~K}$ below the bulk melting point, and it may be that this is what we see in the HRTEM video recordings.

During studies of the surface growth of small $\mathrm{Ru}$ clusters $^{14}$ it has been found that, prior to the first atomic column being added to a $\{111\}$ surface, diffuse, fluctuating contrast was often seen. This has also been found for a $\{100\}$ surface of a gold cluster (cf. Fig. 5). As reported in the first paper on atomic clouds, ${ }^{21}$ it appears to be somewhat ordered, as seen in Figs. 5(a) and (b). The contrast (marked with arrowheads) is more diffuse and weak in (a) compared to (b). When the atoms of the new surface layer [marked with a long arrow in (b)] are almost settled in their positions a weak new "cloud" [marked with an arrowhead in (c)] of atoms for the next layer appears.

There is no doubt that the information from the HRTEM images suggests an initial state of growth (pre-growth) of a 
new surface, while the first atoms approaching the surface will jump around searching for a sufficient number of stable bonds. First, with some critical number of atoms the ordering of columns of atoms will start, with an increasing number of bonds available not only down to the basic surface but also within the new columns. The pre-growth "cloud" of atoms can reach several levels of height before the formation of well ordered columns of one layer starts.

After the "cloudy" pre-growth state, different ordering phenomena of the atoms in the columns start, with almost correct distances from the surface. This stage of growth usually includes apparent column-hopping ${ }^{25}$ in the videosequences. Several mechanisms have been found ${ }^{13.26}$ for different surfaces and elements. Here a new mechanism for a $\{100\}$ surface of a gold cluster is presented (cf. Fig. 6). In this case the pre-growth stage has passed, and in the first image three new atom columns [marked with arrowheads in (a)] are situated in the middle of a (presumably) $6 \times 6$ square surface. The maximum amount of atoms in the top layer in (a) is $3 \times 5$, and when more atoms are incorporated into the surface columns they rearrange the occupancy so that all five columns [marked with arrowheads in (b)] show occupancy, with probably less than a total of 25 atoms. The column on the far right has a very low occupancy $(=1$ atom?) and there is a bridging contrast to the neighbouring column, indicating transport between the two positions during the recording. There is also a contrast concentrated
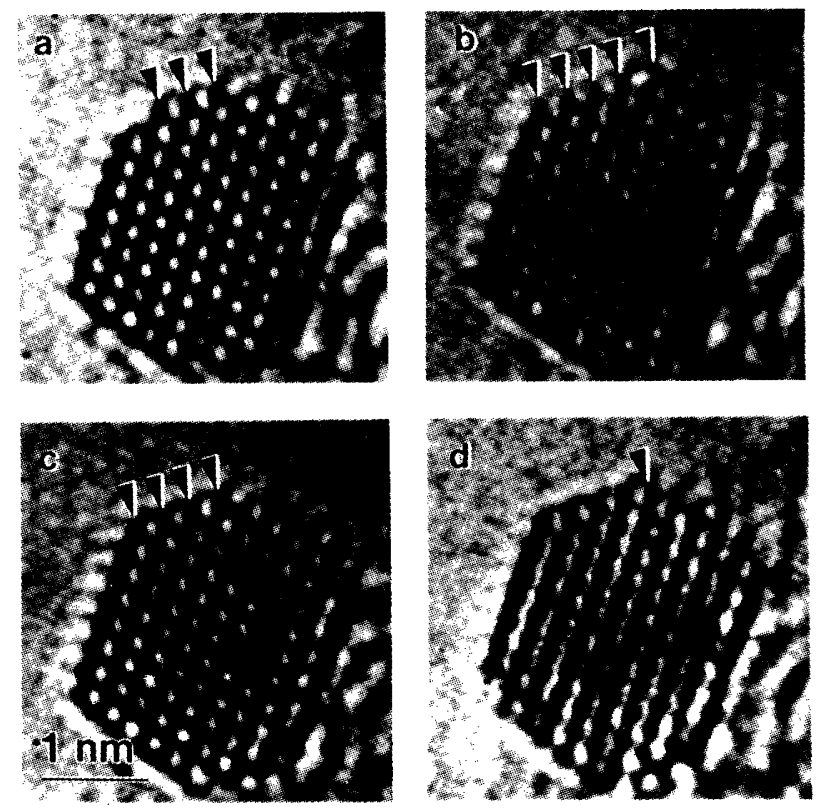

Fig. 6. Single-frame images taken from a few seconds of a video sequence, illustrating one mechanism of adding atoms to a growing $\{100\}$ surface of a gold cluster. In (a) the three first fully occupied columns (marked with arrowheads) containing not more than $3 \times 5$ gold atoms can be seen, and in (b) the atoms are distributed in a moderately filled stage in five columns (marked with five arrowheads) with varying occupancy. Four columns of atoms are marked with arrowheads in (c), and the fifth column (marked with arrowhead) is finally partly filled in (d). half way between. The next image shows how the surface has been rearranged to an occupancy of four columns with strong contrast [marked with four arrowheads in (c)], possibly including $4 \times 5$ atoms. when more than 20 atoms approach the surface the last (fifth) column [marked with an arrowhead in (d)] starts to be filled. The time taken for the sequence presented here is only a few seconds.

Atom hopping during crystal growth. One column of atoms is often seen in HRTEM images of different surfaces of metal clusters. In order to verify the possibility of imaging just one atom added to a surface, image simulations have been made for a model with atom columns of different occupancies. ${ }^{26}$ This simulation indicates that, under Scherzer focus conditions, it should also be possible to find the atom positions in the bulk lattice sites for the outer layer of atoms. It is difficult to estimate the difference in
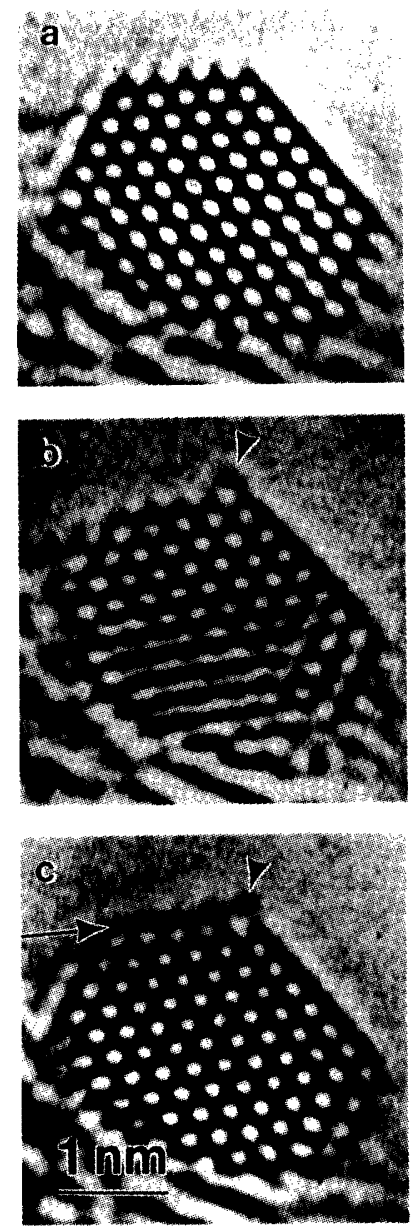

Fig. 7. Single frames from a videosequence of surface profile images showing a $\{100\}$ gold surface projected along [110]. To the clean $\{100\}$ surface in (a) a single column of four atoms or less is added [marked with an arrowhead in (b)]. The surface coordination distances for the single column are slightly too long, and even after the surface has been totally covered with a new layer [marked with a long arrow in (c)] of atoms, the same column [marked with an arrowhead in (c)] keeps its off position. Note the change in contrast in (b) and (c) of the solitary column. 
contrast between, for example, columns with five and three atoms, but there is a difference between five and one atom. The simulation shows a considerable contrast from one atom of gold which it should be possible to see in an experimental image.

An example of a HRTEM image sequence showing a single surface atomic column of varying contrast being added to a $\{100\}$ surface of gold is illustrated in Fig. 7. The first image shows a clean $\{100\}$ surface, and a few frames later a solitary, with somewhat diffuse contrast, atomic column [marked with an arrowhead in (b)] is added to the surface. It seems to be slightly out of its normal lattice site, but later the same column [marked with an arrowhead in (c)] has the same site and weaker contrast. In Fig. 7(c) the entire $\{100\}$ surface has been covered by a new layer of atoms (marked with a long arrow). The new surface layer has diffuse contrast, which is often seen, indicating some disorder during the $1 / 25 \mathrm{~s}$ taken to record the frame. It is possible that the solitary dark contrast, marked with arrowheads in Figs. 7(b) and (c), represent four atoms in projection or less. The $\{100\}$ surface of the cluster in Fig. 7(a) is most likely a $5 \times 5$ square atomic layer (the cluster shape of a cuboctahedron), and the next layer being added will be a $4 \times 4$ layer.

\section{Conclusions}

This paper summarizes the different dynamic features observed on small metal particles using HRTEM. The features include total structural rearrangements, the occurrence of "clouds" of atoms outside the surfaces and localised surface rearrangements, including imaging of single atomic columns.

Twinning in small particles has been shown to appear and disappear during observation, and it is not possible definitely to anneal away twin planes by beam heating. Particles with five-fold symmetry are shown, but to determine their atomic structure exclusively has proved difficult even using image simulations. Atomic level HREM shows that the mechanism of the growth of a new surface layer often includes a diffuse cloud of atoms outside the surface prior to ordering in the expected lattice sites. The sequence of completing the occupancy of atomic columns on a new surface can be followed directly in TV recordings even though it is hard to determine the number of atoms in the observed column. It should also be pointed out that a complication in interpreting the number of atoms is that the time for recording the frame is very long in the world of atoms on the run. The number of atoms in the column can thus vary, and the contrast thus gives merely an idea of the average number of atoms.
Acknowledgements. The cluster project was supported by The Swedish Natural Science Research Council and by the National Energy Administration of Sweden. The availability of the JEM-4000EX electron microscope was made possible through grants from the Alice and Knut Wallenberg Foundation. Prof. G. Schmid and his coworkers are acknowledged for providing us with cluster samples.

\section{References}

1. Ino, S. J. Phys. Soc. Jpn. 27 (1967) 941.

2. Cosslet, V. E., Camps, A. R., Saxton, W. O., Smith, D. J., Nixon, W. C., Ahmed, H., Catto, C. J. D., Cleaver, J. R. A., Smith, K. C. A., Timbs, A. E., Turner, P. W. and Ross, P. M. Nature (London) 281 (1979) 49.

3. Marks, L. D. and Smith, D. J. Nature (London) 303 (1983) 316.

4. Bovin, J.-O. ORDO 16 (1982).

5. Wallenberg, L. R., Bovin, J.-O. and Schmid, G. Surf. Sci. 156 (1985) 256.

6. Iijima, S. and Ichihashi, T. Phys Rev. Lett. 56 (1986) 616.

7. Wallenberg, L. R., Bovin, J.-O. and Smith, D. J. Inst. Phys. Conf. 78 (1986) 481 .

8. Mitome, M.. Tanishiro, Y. and Takayanagi, K. Z. Phys. D 12 (1989) 45.

9. Schmid, G. Struct. Bonding (Berl.) 62 (1985) 52.

10. Schmid, G., Morun, B. and Malm, J.-O. Angew. Chem., Int. Ed. Engl. 28 (1989) 778.

11. O'Keefe, M. A. and Buseck, P. Trans. Am. Cryst. Assoc. 15 (1979) 27.

12. Wallenberg, L. R., Bovin, J.-O., Petford-Long, A. K. and Smith, D. J. Ultramicroscopy 20 (1986) 71.

13. Malm, J.-O., Bovin, J.-O., Petford-Long, A. K. and Smith, D. J. J. Cryst. Growth 89 (1988) 165.

14. Malm, J.-O., Bovin, J.-O., Petford-Long, A. K., Schmid, G. and Klein, N. Angew. Chem., Int. Ed. Engl. 27 (1988) 55.

15. Malm, J.-O. Ultramicroscopy 31 (1989) 143.

16. Ajayan, P. M. and Marks, L. D. Phys. Rev. Lett. 60 (1988) 585.

17. Lidin, S., Andersson, S., Bovin, J.-O., Malm, J.-O. and Terasaki, O., Acta Crystallogr A 45 (1989) FC33.

18. Andersson, S. and Hyde, B. G. J. Solid State Chem. 9 (1974) 92.

19. Hyde, B. G. and Andersson, S. Inorganic Crystal Structures, Wiley, New York 1989.

20. Barlow, W. Nature (London) 29 (1883) 205.

21. Bovin, J.-O., Wallenberg, L. R. and Smith, D. J. Nature (London) 317 (1985) 47.

22. Iijima, S. Arizona State University Centennial Symposium on HREM, Scottsdale, AZ 1985.

23. Murooka, K., Mitome, M., Tanishiro, Y. and Takayanagi, K. J. Vac. Sci. Technol. A8 (1990) 153.

24. Stolze, P., Norskov, J. K. and Landman, V. Phys. Rev. Lett. 61 (1988) 440.

25. Wallenberg, L. R., Bovin, J.-O. and Smith, D. J. Naturwissenschaften 72 (1985) 539.

26. Malm, J.-O. and Bovin, J.-O. Surf. Sci. 200 (1988) 67.

Received November 28, 1990. 